\title{
Dipole response in neutron-rich nuclei within self-consistent approaches us- ing realistic potentials
}

\author{
N. Lo ludice ${ }^{1,2, a}$, F. Knapp 3 , P. Vesely ${ }^{4}$, F. Andreozzi ${ }^{1,2}$, G. De Gregorio ${ }^{1,2}$, and A. Porrino ${ }^{1,2}$ \\ ${ }^{1}$ Dipartimento di Fisica, Università di Napoli Federico II, Italy \\ ${ }^{2}$ INFN Sezione di Napoli, Italy \\ ${ }^{3}$ Institute of Particle and Nuclear Physics, Charles University, Prague, Czech Republic \\ ${ }^{4}$ Institute of Nuclear Physics, Academy of Sciences, Rez, Czech Republic
}

\begin{abstract}
A nucleon-nucleon chiral potential with a corrective density dependent term simulating a three-body force is used in a self-consistent calculation of the dipole strength distribution in neutron-rich nuclei, with special attention to the low-lying spectra associated to the pygmy resonance. A Hartree-Fock-Bogoliubov basis is generated and adopted in Tamm-Dancoff and random-phase approximations and, then, in an equation of motion approach which includes a basis of two-phonon states. The direct use of the mentioned chiral potential improves the description of both giant and pygmy dipole modes with respect to other realistic interactions. Moreover, the inclusion of the two-phonon states induces a pronounced fragmentation of the giant resonance and enhances the density of the low-lying levels in the pygmy region in agreement with recent experiments.
\end{abstract}

\section{Introduction}

Extensive experimental and theoretical studies have been devoted in recent years to the dipole response in neutron rich nuclei. The interest is focused mainly on the dipole strength which appears below the region of the giant dipole resonance (GDR) only in nuclei with neutron excess. These low-lying excitations are interpreted as a manifestation of a soft collective mode, denoted by pygmy dipole resonance (PDR), induced by a translational oscillation of the excess neutrons against a $\mathrm{N}=\mathrm{Z}$ core.

Experimental evidence for such an additional strength below the GDR has been gained in stable and unstable neutron rich nuclei. A comprehensive and updated list of references can be found in a recent review [1]. We mention only few of them.

An appreciable dipole strength just above the neutron decay threshold was extracted through Coulomb scattering in inverse kinematic in tin isotopes around the unstable ${ }^{132} \mathrm{Sn}$ [2]. In stable nuclei, dense discrete spectra of dipole states were detected in $\left(\gamma, \gamma^{\prime}\right)$ [3-9] and in $\left(\alpha, \alpha^{\prime} \gamma\right)$ experiments [10-12]. Moreover, fairly complete dipole spectra below and above the neutron decay threshold were produced with great accuracy through inelastic proton scattering experiments $[13,14]$.

The structure of these low-energy states was investigated in several theoretical approaches. A fairly exhaustive list of references can be found in Refs. $[1,15,16]$. Most investigations were carried out within phenomenological mean field approaches embedded in energy density

\footnotetext{
a e-mail: loiudice@na.infn.it
}

functionals derived from Skyrme forces [17] or from relativistic meson-nucleon Lagrangians [18].

Following an alternative route proposed recently [19], we carried out self-consistent calculations using directly realistic potentials derived from nucleon-nucleon (NN) forces. We have first adopted a $V_{\text {lowk }}$ potential derived from the CD-Bonn NN interaction to generate a HartreeFock-Bogoliubov (HFB) basis and, then, used such a basis to solve the eigenvalue equations in both (quasi-particle) Tamm-Dancoff ((Q)TDA) and random-phase approximations ((Q)RPA). We could therefore perform a systematic study of the dipole response in several chains of neutron rich isotopes [20]. As in [19], however, we found necessary to add a corrective density dependent piece in order to get single-particle spectra sufficiently compressed so as to reproduce the main peak of the experimental GDR. Using such a modified Hamiltonian, we obtained spectra qualitatively consistent with experiments for all light, medium mass and heavy isotopes [20].

More recently, we performed analogous selfconsistent (Q)TDA and (Q)RPA studies by making direct use of a recently determined chiral potential, whose parameters were optimized so as to minimize the effects of the three-body forces [21]. This potential was adopted for ${ }^{132} \mathrm{Sn}$ in [22] and found to yield a much more compact single particle spectrum compared to $V_{\text {lowk }}$ and, consequently, to produce a dipole strength distribution much closer to the region of observation of the GDR peak. It was, nonetheless, still necessary to add the same density dependent term, though with a weaker strength, in order to reproduce the centroid of the resonance. The potential, 

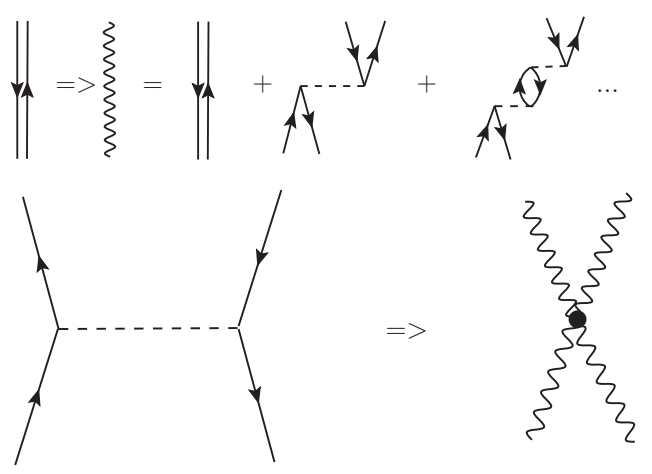

Figure 1. From TDA (wavy line) to EMPM.

so modified, reproduces satisfactorily the gross features of both GDR and PDR.

(Q)TDA and (Q)RPA, however, were unable to describe the fine structure of the two resonances. So, we went beyond the mean field approximation and adopted the equation of motion phonon method (EMPM) [23-25] to study the dipole response within a space spanned by one plus two-phonon basis states [22]. The two phonons enhanced enormously the fragmentation of the dipole strength in both GDR and PDR regions, in better agreement with experiments. We report here on the findings of these investigations.

\section{Brief description of the method}

The EMPM gives a general prescription for solving the nuclear eigenvalue problem in a multiphonon space, the phonons being generated in TDA. Its first goal is to generate, within a given $n$-phonon subspace, a basis of states $|n ; \beta\rangle$ of energies $E_{\beta}$ having the form

$$
|n ; \beta\rangle=\sum_{\lambda \alpha} C_{\lambda \alpha}^{\beta}\left\{O_{\lambda}^{\dagger} \times|n-1, \alpha\rangle\right\}^{\beta} .
$$

Here

$$
O_{\lambda}^{\dagger}=\sum_{p h} c_{p h}^{\lambda}\left(a_{p}^{\dagger} \times b_{h}\right)^{\lambda}
$$

is the TDA particle-hole $(\mathrm{p}-\mathrm{h})$ phonon operator of energy $E_{\lambda}$ acting on the $(n-1)$-phonon states $|n-1, \alpha\rangle$ of energies $E_{\alpha}$. These phonons are generated by solving the TDA eigenvalue equations

$$
\sum_{p h p^{\prime} h^{\prime}}\left[A_{p h p^{\prime} h^{\prime}}^{\lambda}-E_{\lambda} \delta_{p p^{\prime}} \delta_{h h^{\prime}}\right] c_{p^{\prime} h^{\prime}}^{\lambda}=0 .
$$

$A$ is the TDA matrix

$$
A_{p h p^{\prime} h^{\prime}}^{\lambda}=\left(\epsilon_{p}-\epsilon_{h}\right) \delta_{p p^{\prime}} \delta_{h h^{\prime}}+(-)^{p-h+\lambda} F_{h p p^{\prime} h^{\prime}}^{\lambda}
$$

where

$$
F_{r t s q}^{\lambda}=\sum_{\sigma}[\sigma](-)^{r+q-\lambda-\sigma} W(r s t q ; \sigma \lambda) V_{r s t q}^{\sigma}
$$

and $W$ are Racah coefficients. The EMPM procedure starts with the equations of motion

$$
\begin{array}{r}
\left\langle n, \beta\left\|\left[H, O_{\lambda}^{\dagger}\right]\right\| n-1, \alpha\right\rangle= \\
\left(E_{\beta}-E_{\alpha}\right)\left\langle n, \beta\left\|O_{\lambda}^{\dagger}\right\| n-1, \alpha\right\rangle .
\end{array}
$$

After expanding the commutator and expressing $\left(a_{p}^{\dagger} \times b_{h}\right)^{\lambda}$ in terms of the phonon $O_{\lambda}^{\dagger}$ operator upon inversion of Eq. (2), we obtain [25] the generalized eigenvalue equation

$$
\begin{aligned}
\sum_{\lambda^{\prime} \alpha^{\prime} \lambda_{1} \alpha_{1}}[ & \left.\mathcal{A}^{\beta}\left(\lambda \alpha, \lambda_{1} \alpha_{1}\right)-E_{\beta} \delta_{\lambda_{1} \lambda} \delta_{\alpha_{1} \alpha}\right] \\
& \times \mathcal{D}^{\beta}\left(\lambda_{1} \alpha_{1}, \lambda^{\prime} \alpha^{\prime}\right) C_{\lambda^{\prime} \alpha^{\prime}}^{\beta}=0,
\end{aligned}
$$

where

$$
\begin{array}{r}
\mathcal{D}^{\beta}\left(\lambda_{1} \alpha_{1} ; \lambda^{\prime} \alpha^{\prime}\right)= \\
{\left[\left\langle n-1, \alpha_{1}\right| \times O_{\lambda_{1}}\right]_{\beta}\left[O_{\lambda^{\prime}}^{\dagger} \times\left|n-1, \alpha^{\prime}\right\rangle\right]_{\beta}}
\end{array}
$$

is the metric matrix and $\mathcal{A}^{\beta}$ is a matrix of the simple structure

$$
\mathcal{A}^{\beta}\left(\lambda \alpha, \lambda^{\prime} \gamma\right)=\left(E_{\lambda}+E_{\alpha}\right) \delta_{\lambda \lambda^{\prime}} \delta_{\alpha \gamma}+\mathcal{F}_{\lambda \alpha \lambda^{\prime} \gamma}^{\beta}
$$

Here

$$
\mathcal{F}_{\lambda \alpha \lambda^{\prime} \gamma}^{\beta}=\sum_{\sigma} W\left(\beta \lambda^{\prime} \alpha \sigma ; \gamma \lambda\right) \mathcal{V}_{\lambda \alpha, \lambda^{\prime} \gamma}^{\sigma}
$$

and

$$
\mathcal{V}_{\lambda \alpha, \lambda^{\prime} \gamma}^{\sigma}=\sum_{r s t q} \rho_{\lambda \lambda^{\prime}}\left([q \times t]^{\sigma}\right) F_{q t r s}^{\sigma} \rho_{\alpha \gamma}^{(n)}\left([r \times s]^{\sigma}\right),
$$

$\rho_{\lambda \lambda^{\prime}}$ and $\rho_{\alpha \alpha^{\prime}}^{(n)}$ being, respectively, the $n=1$ (TDA) and the $n$-phonon $(n>1)$ density matrices both defined as

$$
\rho_{\alpha \alpha^{\prime}}^{(n)}\left([r \times s]^{\sigma}\right)=\left\langle n ; \alpha^{\prime}\left\|\left[a_{r}^{\dagger} \times b_{s}\right]^{\sigma}\right\| n ; \alpha\right\rangle .
$$

The analogy with the TDA eigenvalue problem is to be noticed. The phonon matrix $\mathcal{A}^{\beta}\left(\lambda \alpha, \lambda^{\prime} \alpha^{\prime}\right)$ is formally identical to the TDA matrix $A^{\lambda}\left(p h ; p^{\prime} h^{\prime}\right)$ given by Eq. (4). The phonon energies $\left(E_{\lambda}+E_{\alpha}\right)$ correspond to the TDA p-h energies, while the phonon-phonon interaction $\mathcal{F}^{\beta}$ replaces the TDA p-h interaction $F^{\lambda}$. A picture of such a correspondence is given in Fig. 1.

Eq. (7) represents the eigenvalue equation in the overcomplete basis $\left\{O_{\lambda}^{\dagger} \times|n-1, \alpha\rangle\right\}^{\beta}$. The redundant states are eliminated by the procedure outlined in $[23,24]$, based on the Cholesky decomposition method.

Since recursive formulas hold for all quantities entering $\mathcal{A}$ and $\mathcal{D}$, the eigenvalue equations are solved iteratively starting from the TDA phonons and, thereby, yield a set of orthonormal multiphonon basis states $\{|0\rangle$, | $1, \lambda\rangle, \cdots|n, \alpha\rangle \ldots\}$. In such a basis, the Hamiltonian matrix is composed of a sequence of diagonal blocks, one for each $n$, mutually coupled by off-diagonal terms $\left\langle n^{\prime}|H| n\right\rangle$ which are non vanishing only for $n^{\prime}=n \pm 1, n \pm 2$ and are computed by means of recursive formulas. A matrix of such a simple structure can be easily diagonalized. 

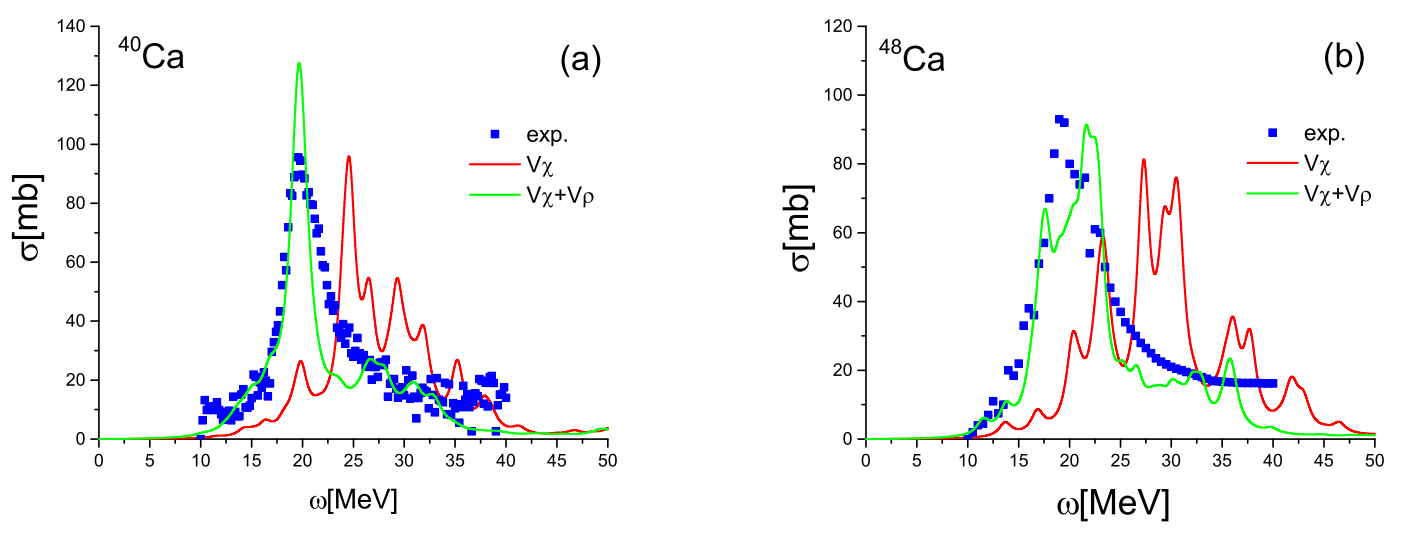

Figure 2. TDA dipole cross section computed for ${ }^{40} \mathrm{Ca}$ and ${ }^{48} \mathrm{Ca}$ using $V_{\chi}$ only and $V_{\chi}+V_{\rho}$.

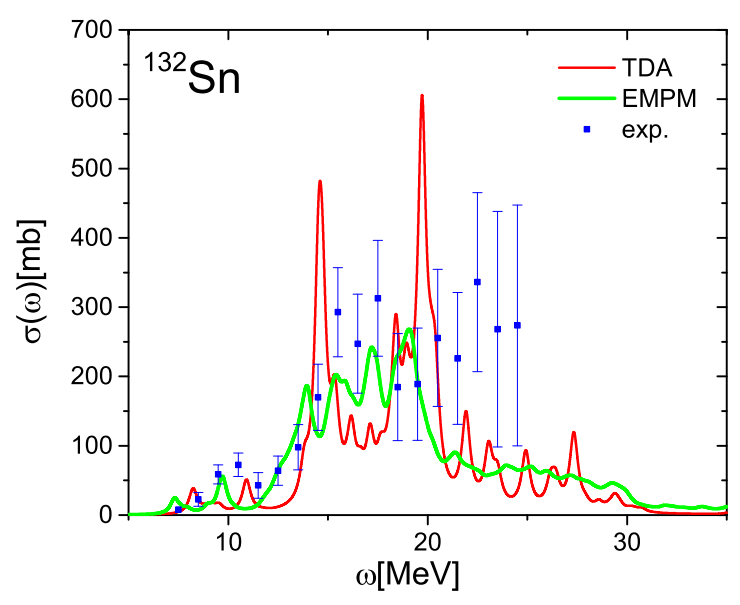

Figure 3. EMPM and TDA versus experimental cross sections in ${ }^{132} \mathrm{Sn}$. A Lorentzian width $\Delta=0.5 \mathrm{MeV}$ was used to compute the cross sections.

\section{Calculations and results}

We used an intrinsic Hamiltonian obtained by subtracting the center of mass $(\mathrm{CM})$ kinetic energy $T_{C M}$ from the shell model kinetic operator. Its final form is composed of the usual kinetic energy operator $T$ with an effective nucleon mass $m A /(A-1)$ plus a potential $V=T_{2}+V_{2}$. This includes a a two-body kinetic piece

$$
T_{2}=-\frac{1}{2 m A} \sum_{i \neq j} \vec{p}_{i} \cdot \vec{p}_{j}
$$

plus the potential

$$
V_{2}=V_{\chi}+V_{\rho}
$$

$V_{\chi}$ is the $N N$ optimized chiral potential denoted by $N N L O_{\text {opt }}$ in Refs. [21, 22], while $V_{\rho}$ is a corrective repulsive, density dependent, two-body potential with a coupling constant $C_{\rho}$. This force, introduced to simulate a three-body contact force [26], improves the description of bulk properties in closed-shell nuclei [27] and yields more realistic single-particle spectra and multipole-nuclear responses [19, 20].

We performed a HFB calculation in a configuration space which includes 13 harmonic oscillator major shells, up to the principal quantum number $N_{\max }=12$. The self-consistent basis was then used to compute the dipole strength distribution in both (Q)TDA and (Q)RPA. In a fully self-consistent (Q)RPA, the $1^{-}$spurious state lies at zero excitation energy and collects the total strength induced by the CM. Numerically, it is very difficult to achieve a complete decoupling from the physical intrinsic states unless a huge configuration space is adopted [19]. In our space, the energy of the lowest $1^{-}$(Q)RPA spurious state acquires a small imaginary component of the order $\sim 0.1 \mathrm{MeV}$. Small spurious admixtures are therefore present in the strength distribution [20]. A qualitatively similar result is obtained for (Q)TDA. In this case, the lowest $1^{-}$, which gets almost the whole CM strength, lies at slightly negative, $\omega_{1^{-}}^{(1)} \sim-0.1 \mathrm{MeV}$.

In (Q)TDA, however, the case of interest to us, we obtain completely spurious free states by adopting the Gramm-Schmidt method to orthogonalize the particlehole (or two quasi-particle) basis states to the CM state [20]. The use of (Q)TDA instead of (Q)RPA does not imply any restriction. The two approaches yield almost identical dipole spectra in all chains of isotopes [20,22].

A comparative analysis of the spectra plotted in Refs. $[22,25]$ shows that $V_{\chi}$ yields single-particle spectra considerably more compressed than $V_{\text {lowk }}$. This compression is more consistent with experiments and improves the (Q)TDA and (Q)RPA descriptions of the dipole response. In ${ }^{132} \mathrm{Sn}$, for instance, it pushes the main peak of the dipole strength distribution down to $\sim 23 \mathrm{MeV}$ [22], an energy much lower than the peak energy, $\sim 45 \mathrm{MeV}$, obtained with $V_{\text {lowk }}[20]$.

The downward shift produced by such a pronounced compression is not sufficient to push the peak down to the experimental one which lies at $\sim 16 \mathrm{MeV}$ in ${ }^{132} \mathrm{Sn}$. Hence the need of adding the density dependent term $V_{\rho}$, though with a weaker strength. We used for all nuclei of all regions the coupling constant $C_{\rho} \sim 1000 \mathrm{MeV} \mathrm{fm}^{6}$, four 


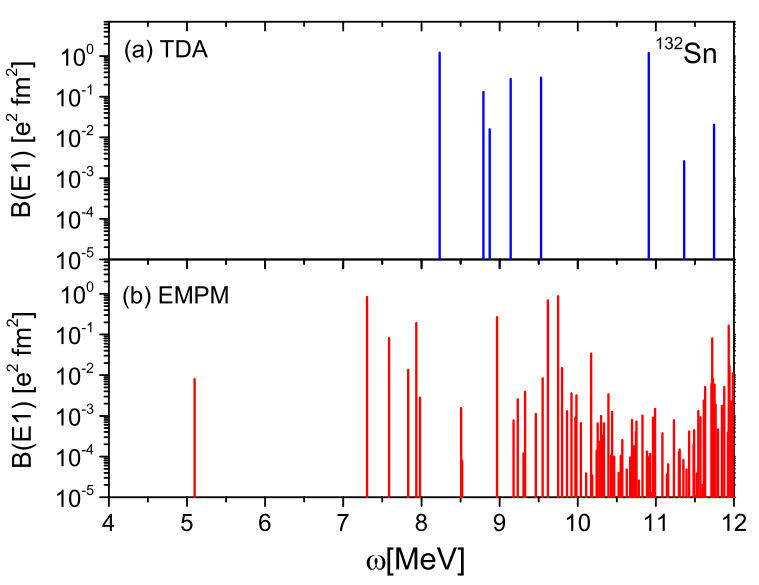

Figure 4. EMPM versus TDA low-lying $E 1$ spectra in ${ }^{132} \mathrm{Sn}$.

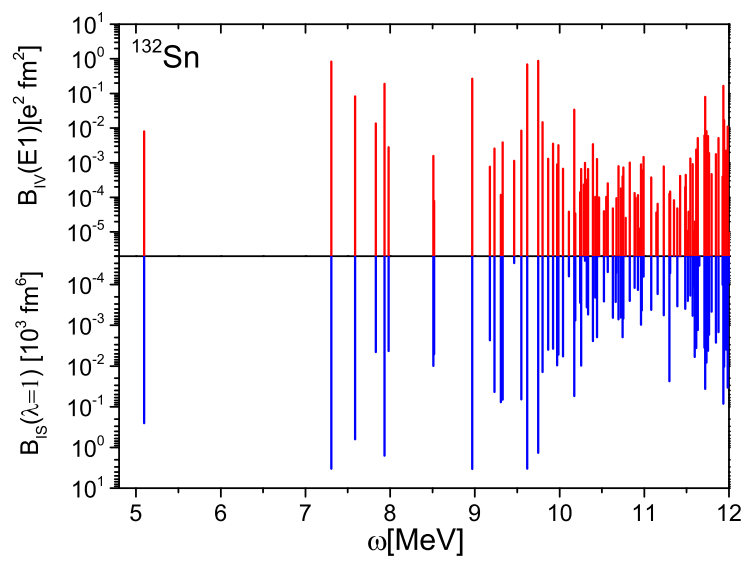

Figure 5. Low-lying isoscalar versus isovector dipole spectra in ${ }^{132} \mathrm{Sn}$ computed in EMPM

times smaller than the $V_{\rho}$ strength $\left(C_{\rho} \sim 4200 \mathrm{MeV} \mathrm{fm}^{6}\right)$ used in the case of $V_{\text {lowk }}$ [20]. By using the potential so corrected, we get a fair agreement between theoretical and experimental cross sections (Fig. 2).

Both (Q)TDA and (Q)RPA describe properly the gross features of GDR and PDR. To reproduce their fine structure it is necessary to go beyond the mean field approximation. This was done for ${ }^{132} \mathrm{Sn}$ by using the EMPM [22].

In the present EMPM calculation, the $1^{-}$states are obtained by diagonalizing the Hamiltonian in a space spanned by one- $\left(\left|\lambda, 1^{-}\right\rangle\right)$plus two-phonon $\left(\left|(n=2) \alpha, 1^{-}\right\rangle\right)$ basis states. The latter states are generated in a subspace which includes the states $\left|\left(\lambda_{1} \times \lambda_{2}\right)^{1^{-}}\right\rangle \equiv\left\{O_{\lambda_{1}}^{\dagger} \times\left|\lambda_{2}\right\rangle\right\}^{1^{-}}$of energies $E_{\lambda_{1}}+E_{\lambda_{2}} \leq 30 \mathrm{MeV}$.

The inclusion of the two-phonon states has a strong damping effect on the response. Compared to TDA, the EMPM cross section is severely quenched and reshaped due to the one- to two-phonon coupling (Fig. 3). It has a smoother behavior and follows more closely the experimental points [2].

Both TDA and EMPM calculations yield a small peak in the cross section around $\sim 10 \mathrm{MeV}$, fairly close in position and height to the one at $\sim 9.8 \mathrm{MeV}$ observed exper- imentally [2]. The EMPM strength collected by the lowlying states up to $\sim 11 \mathrm{MeV}$ exhausts $\sim 5.8 \%$ of the TRK sum rule. This value is within the error of the measured fraction $4(3) \%$ [2].

The two spectra, however, are quite different. Fig. 4 shows that, compared to TDA, the EMPM low-lying spectrum is much more dense. The analysis of the wavefunctions and the corresponding transition densities suggests the pygmy nature of the most strongly excited states[22].

Combined $\left(\gamma, \gamma^{\prime}\right)$ and $\left(\alpha, \alpha^{\prime} \gamma\right)$ experiments on the open shell ${ }^{124} \mathrm{Sn}[11,12,28]$ found that the low-lying dipole strength splits into an upper sector composed of isovector transitions promoted by $\gamma$ 's and a lower region of levels populated by both $\alpha$ 's and $\gamma$ 's. Only these low-lying peaks form therefore the PDR. We have computed an analogous theoretical analysis within the EMPM for ${ }^{132} \mathrm{Sn}$ and did not find such a splitting. Practically all the low-lying levels get excited by both isoscalar and isovector probes (Fig. 5).

\section{Conclusions}

An important aspect emerging from the investigation of the dipole strength evaluated by direct use of the NN interaction is the sensitivity of the response to different NN nuclear forces. This property may be exploited to test different NN potentials and to suggest a way of improving their parametrization.

In our case, the optimized chiral potential $V_{\chi}$ came out to yield much more realistic HF spectra compared to other realistic potentials and to improve drastically the description of the dipole response. It represents, therefore, a promising first step of the ambitious project of offering a self-consistent and reliable study of the dipole as well as other collective modes in stable and unstable nuclei starting directly from the $\mathrm{NN}$ interaction.

We must point out that this first step is not sufficient. We have seen, in fact, that the chiral potential alone is not able to fill completely the gap between theory and experiments. The phenomenological density dependent term we added in order to reproduce the global properties of the GDR and PDR resonances suggests that the potential should be rendered more repulsive either by a better parametrization or a weak three-body force.

The present study has also pointed out the crucial role of complex configurations. Indeed, the two-phonon configurations have a damping and smoothing action which yields a GDR cross section close to the experimental one in shape and magnitude. They also enhance enormously the density of levels at low energy thereby producing discrete spectra comparable with the ones produced in $\left(\gamma, \gamma^{\prime}\right)$ and $\left(p, p^{\prime}\right)$ experiments. The calculation on ${ }^{132} \mathrm{Sn}$, however, does not produce a splitting between a low region of isoscalar transitions of pygmy nature and an upper region of isovector transitions as found experimentally for open shell tin isotopes. This difference may be due to the double magic nature of ${ }^{132} \mathrm{Sn}$ or to an insufficient accuracy of the calculation.

This, indeed, can still be upgraded significantly. It is desirable to improve further the $\mathrm{NN}$ potential so as to 
generate more accurate single-particle energies, which the low-energy dipole levels are quite sensitive to. It is also necessary to include the three-phonon states. The coupling of these configurations to the one-phonon states is strong and comparable to the coupling of the two-phonon states to the HF vacuum. By including both couplings, it would be possible to take full advantage of the ground state correlations and therefore obtain a rather complete description of the dipole response.

\section{Acknowledgements}

This work was partially supported by the Czech Science Foundation (Project No. P203-13-07117S)

\section{References}

[1] D. Savran, T. Aumann, A. Zilges, Progress in Particle and Nuclear Physics 70, 210 (2013)

[2] P. Adrich, A. Klimkiewicz, M. Fallot, K. Boretzky, T. Aumann, D. Cortina-Gil, U. D. Pramanik, T. W. Elze, H. Emling, H. Geissel, M. Hellström, K. L. Jones, J. V. Kratz, R. Kulessa, Y. Leifels, C. Nociforo, R. Palit, H. Simon, G. Surówka, K. Sümmerer and W. Waluś (LAND-FRS Collaboration), Phys. Rev. Lett. 95, 132501 (2005)

[3] A. Zilges, S. Volz, M. Babilon, T. Hartmann, P. Mohr, K. Vogt, Physics Letters B 542, 43 (2002)

[4] T. Hartmann, M. Babilon, S. Kamerdzhiev, E. Litvinova, D. Savran, S. Volz, A. Zilges, Phys. Rev. Lett. 93, 192501 (2004)

[5] R. Schwengner, G. Rusev, N. Benouaret, R. Beyer, M. Erhard, E. Grosse, A.R. Junghans, J. Klug, K. Kosev, L. Kostov et al., Phys. Rev. C 76, 034321 (2007)

[6] J. Isaak, D. Savran, M. Fritzsche, D. Galaviz, T. Hartmann, S. Kamerdzhiev, J.H. Kelley, E. Kwan, N. Pietralla, C. Romig et al., Phys. Rev. C 83, 034304 (2011)

[7] D. Savran, M. Elvers, J. Endres, M. Fritzsche, B. Löher, N. Pietralla, V.Y. Ponomarev, C. Romig, L. Schnorrenberger, K. Sonnabend et al., Phys. Rev. C 84, 024326 (2011)

[8] V. Derya, D. Savran, J. Endres, M. N. Harakeh, H. Hergert, J.H. Kelley, P. Papakonstantinou, N. Pietralla, V.Y. Ponomarev, R. Roth, G. Rusev et al., Phys. Lett. B 730, 288 (2014)

[9] B. Özel-Tashenov, J. Enders, H. Lenske, A.M. Krumbholz, E. Litvinova, P. von Neumann-Cosel, I. Poltoratska, A. Richter, G. Rusev, D. Savran et al., Phys. Rev C 90, 024304 (2014)

[10] D. Savran, M. Babilon, A.M. van den Berg, M.N. Harakeh, J. Hasper, A. Matic, H. J. Wörtche and A. Zilges, Phys. Rev. Lett. 97, 172502 (2006)
[11] J. Endres, E. Litvinova, D. Savran, P.A. Butler, M.N. Harakeh, S. Harissopulos, R.D. Herzberg, R. Krücken, A. Lagoyannis, N. Pietralla et al., Phys. Rev. Lett. 105, 212503 (2010)

[12] J. Endres, D. Savran, P.A. Butler, M.N. Harakeh, S. Harissopulos, R.D. Herzberg, R. Krücken, A. Lagoyannis, E. Litvinova, N. Pietralla et al., Phys. Rev. C 85, 064331 (2012)

[13] A. Tamii, I. Poltoratska, P. von Neumann-Cosel, Y. Fujita, T. Adachi, C.A. Bertulani, J. Carter,M. Dozono, H. Fujita, K. Fujita et al., Phys. Rev. Lett. 107, 062502 (2011)

[14] I. Poltoratska, P. von Neumann-Cosel, A. Tamii, T. Adachi, C.A. Bertulani, J. Carter, M. Dozono, H. Fujita, K. Fujita, Y. Fujita et al., Phys. Rev. C 85, 041304 (2012)

[15] N. Paar, D. Vretenar, E. Khan, G. Colò, Report on Progress in Physics 70, 691 (2007)

[16] N. Paar, Journal of Physics G: Nuclear and Particle Physics 37, 064014 (2010)

[17] M. Bender, P. H. Heenen and P. G. Reinhard, Rev. Mod. Phys. 75, 121 (2003)

[18] D. Vretenar, A. V. Afanasjev, G. A. Lalazissis and P. Ring, Phys. Rep. 409, 101 (2005)

[19] H. Hergert, P. Papakonstantinou and R. Roth, Phys. Rev. C 83, 064317 (2011)

[20] D. Bianco, F. Knapp, N. Lo Iudice, P. Veselý, F. Andreozzi, G. De Gregorio and A. Porrino, J. of Phys. G: Nucl. and Part. Phys. 41, 025109 (2014)

[21] A. Ekström, G. Baardsen, C. Forssén, G. Hagen, M. Hjorth-Jensen, G. R. Jansen, R. Machleidt, W. Nazarewicz, T. Papenbrock, J. Sarich and S. M. Wild, Phys. Rev. Lett. 110, 192502 (2013)

[22] F. Knapp, N. Lo Iudice, P. Veselý, F. Andreozzi, G. De Gregorio and A. Porrino, Phys. Rev. C 90, 014310 (2014)

[23] F. Andreozzi, F. Knapp, N. Lo Iudice, A. Porrino and J. Kvasil, Phys. Rev. C 75, 044312 (2007)

[24] F. Andreozzi, F. Knapp, N. Lo Iudice, A. Porrino and J. Kvasil, Phys. Rev. C 78, 054308 (2008)

[25] D. Bianco, F. Knapp, N. Lo Iudice, F. Andreozzi and A. Porrino, Phys. Rev. C 85, 014313 (2012)

[26] M. Waroquier, K. Heyde and H. Vincx, Phys. Rev. C 13, 1664 (1976)

[27] A. Günther, R. Roth, H. Hergert and S. Reinhardt, Phys. Rev. C 82, 024319 (2010)

[28] K. Govaert, F. Bauwens, J. Bryssinck, D. De Frenne, E. Jacobs, W. Mondelaers, L. Govor and V. Y. Ponomarev, Phys. Rev. C 57, 2229 (1998) 
